\begin{tabular}{|c|l|}
\hline Title & Topological manifestations of surface roughening collapse in Langmuir monolayers \\
\hline Author(s) & Hatta, E.; Nagao, J. \\
\hline Citation & $\begin{array}{l}\text { Physical Review E, 67, O41604 } \\
\text { https://doi.org/10.1103/PhysRevE.67.041604 }\end{array}$ \\
\hline Issue Date & 2003-04.29 \\
\hline Doc URL & http://hdl.handle.net/2115/5714 \\
\hline Rights & ○2003 The A merican Physical Society \\
\hline Type & article \\
\hline File Information & PRE67.pdf \\
\hline
\end{tabular}

Instructions for use 


\title{
Topological manifestations of surface-roughening collapse in Langmuir monolayers
}

\author{
E. Hatta* \\ Nanoelectronics Laboratory, Graduate School of Engineering, Hokkaido University, Sapporo 060-8628, Japan
}

J. Nagao

Materials Division, Hokkaido National Industrial Research Institute, AIST, MITI, 2-17-2-1 Tsukisamu-Higashi, Toyohira, Sapporo 062-8517, Japan

(Received 6 September 2002; published 29 April 2003)

\begin{abstract}
A transition from surface-roughening collapse to a random network collapse has been investigated in fatty acid Langmuir monolayers. In contrast to the random network crack pattern, the surface-roughening crack pattern grows on a much longer time scale and on a much shorter length scale. A change in the isotherm from a surface pressure "spike" to a "plateau" occurs at the transition. In the surface-roughening regime, the pattern is manifested by the emergence of a three-dimensional (3D) disordered stripe phase with locally aligned, anisotropic stripe clusters. The individual stripes coalesce less with each other even at a late stage of the collapse process and there is a characteristic length for the stripe width and the nearest neighbor spacing. The pattern includes a high density of topological defects such as single open ends, twin open ends, branches, and loops. The effects of shear deformation on the observed patterns are discussed.
\end{abstract}

DOI: 10.1103/PhysRevE.67.041604

\section{INTRODUCTION}

Insoluble monomolecular films at the air-water interface (Langmuir monolayers) are of considerable interest for the study of two-dimensional phase (2D) transitions and critical phenomena, wetting, interfacial microrheology, membrane physics, etc. [1]. They exhibit a variety of mesophases such as liquid expanded(LE) and liquid condensed (LC) phases, which intervene between the two-dimensional gas and solid phases, as shown by grazing incidence $\mathrm{x}$-ray diffraction (GIXD) studies [2,3] as well as the various mesoscopic textures observed by polarized fluorescence microscopy $[4,5]$ and Brewster angle microscopy [6,7].

Besides transitions between two-dimensional phases, there is another class of phase transitions in Langmuir monolayers, in which the monolayer explores the third dimension perpendicular to the water surface upon monolayer overcompression. A deep insight into such two-dimensional to threedimensional collapse transitions is essential for understanding the mechanism of the formation of lipid bilayers or higher structures such as vesicles and folds [8] and further to understand the function of complex natural surfactant systems [9]. Macroscopically a collapse transition is manifested in the isotherm by the appearance of either a sharp surface pressure drop ("spike") or a plateau only at the collapse surface pressure $\pi_{c}$. The magnitude of $\pi_{c}$ reflects the degree of monolayer stability against the collapse and depends on the in-plane interactions in the monolayer as well as on the interaction of the monolayer with the aqueous subphase. Smith and Berg considered the "slow collapse" mechanism by nucleation and its subsequent growth [10]. Generally, if the surface pressure exceeds the equilibrium spreading pressure (ESP), the pressure at which the monolayer is in equilibrium with the bulk phase, the monolayer becomes metastable. As the pressure is increased further, the barrier to

*Electronic address: hatta@nano.eng.hokudai.ac.jp
PACS number(s): 68.18.-g, 62.20.Mk, 46.50.+a

nucleation decreases and the formation of 3D phase critical nuclei becomes more probable. Once critical nuclei have formed, they grow into the bulk phase. It is possible that other collapse modes will occur at higher pressures. The possibility of the formation of an organized trilayer (which is often referred to as the "Ries mechanism" [11]) has been discussed with reference to the isotherms [12] and atomic force microscopy studies [13]. Recently intensive studies of 2D-3D collapse transitions have been carried out with a combination of microscopy and video imaging. The formation of twisted ribbons was observed on both the water surface [14] and the solid substrate [15]. Global folding and buckling can occur in the collapse process of certain monolayers. Three kinds of monolayer collapse mechanism, i.e., the formation of reversible, giant folds and long multiple folds as well as slow collapse were found in 2-hydroxytetracosanoic acid monolayers [16]. Gopal and Lee observed that collapse occurs via folding and vesiculation in DPPC (dipalmitoylphosphatidylcholine) and POPG (palmitoyloleoylphosphatidylglycerol) mixed monolayers and that the transition between the collapse modes resembles an equilibrium first-order phase transition [17]. The collapse modes of stearic acid monolayers upon overcompression were classified into three types of irreversible fracture depending on the degree of head-group ionization of amphiphilic molecules: (1) surface roughening, (2) a random crack network, and (3) anisotropic cracking [18]. The latter two crack morphologies were found to be accompanied by characteristic evolutions; the occurrence of individual cracks in a random crack network pattern is instantaneous, while anisotropic cracking follows a slow dynamics [19]. There are experimental evidences of buckling from light-scattering [20], GIXD [21], and fluorecence microscopy studies [22]. Although much has been reported on the formation of globally collapsed structures as outlined above, there have been few reports on how monolayers undergo a transition to the bulk phase in the slow collapse regime.

In this study we investigate the topological characteristics 


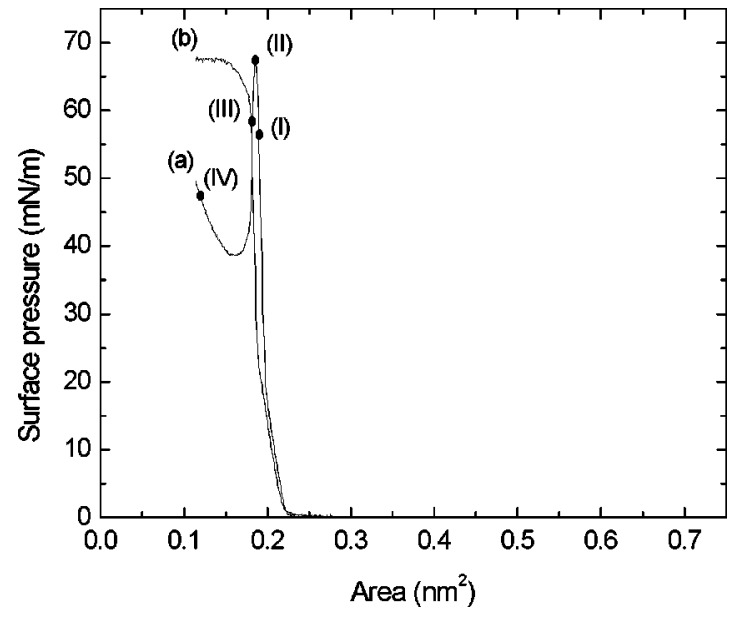

FIG. 1. $\pi-A$ isotherms for stearic acid Langmuir monolayers at (a) $p \mathrm{H}=7.5$ and (b) $p \mathrm{H}=7.8$. The transition from a surface pressure "spike" to a "plateau" is clearly seen.

of the collapse pattern of Langmuir monolayers in the slow collapse regime. This study includes measurements of $\pi$ $-A$ isotherms and imaging by phase contrast microscopy. The creation of a partially aligned, disordered 3D stripe phase is found in this regime. This pattern is characterized by eightfold rotational symmetry and by the prevalence of high density of topological defects as well as the existence of a characteristic length for the stripe width and the spacing between the stripes. The significance of shear deformation on the growth of the stripe phase is stressed.

\section{EXPERIMENTAL SECTION}

Monolayers of stearic acid [octadecanoic acid, C18, 99\% pure, Sigma Chemicals] dissolved in $0.5 \mathrm{mMol} n$-hexane (99\% pure, Kanto Chemicals) were spread onto a subphase (Millipore Mill-Q system filtered water, $18.0 \mathrm{M} \Omega \mathrm{cm}$ ). All experiments were performed at $20.0 \pm 0.2{ }^{\circ} \mathrm{C}$. The $p \mathrm{H}$ value of the subphase was adjusted with $\mathrm{NaHCO}_{3}$. These materials were used without further purification. It has been established that phase contrast microscopy (PCM) is essential for following collapse morphologies and their dynamics [23]. Cracks in the monolayer at the air-water interface were monitored via three-dimensional aggregates protruding from the cracks using phase contrast microscopy (NIKON, OPTIPHOT-2) equipped with a charge-coupled device camera (Hamamatsu, C2400-77H) followed by an image processor (Hamamatsu, DVS-3000). The incident light was trans- mitted through the bottom of a glass trough, which was equipped with a microscope stage. The crack evolution was recorded at a frequency of 30 frames/s while constantly monitoring the $\pi-A$ isotherms. The width of the trough used was $10 \mathrm{~cm}$ and monolayers were compressed at a low barrier speed $(1 \mathrm{~cm} / \mathrm{min})$ to diminish the kinetic effect of barrier compression. This allowed the identification of the effects of head group ionization on the isotherms and the morphologies of the crack modes.

\section{RESULTS AND DISCUSSION}

Figure 1 shows $\pi-A$ isotherms for stearic acid Langmuir monolayers at $p \mathrm{H}=7.5$ and 7.8. A definite transition from a surface pressure spike to a plateau is seen in the collapse region of the isotherms with increasing $p \mathrm{H}$, although both the isotherms exhibit similar behavior below the superliquid phase. Typical crack patterns in the later stages of the collapse process are shown in Fig. 2 for the same $p \mathrm{H}$ values. The dark and bright regions in the images correspond to the 3D collapse structure and the coexisting monolayer, respectively. Therefore, the film after collapse is regarded as one in which a monolayer coexists with multilayered or microcrystalline 3D structures. A transition from a surface roughening (the emergence of granular or microcrystalline 3D structures) to random network cracking occurs, as seen in the PCM images. It is noteworthy that the scale over which cracks develop is quite different for these two.

Figure 3 shows the evolution of a surface-roughening collapse pattern upon monolayer overcompression. Each image was taken at the corresponding points (i.e., I, II, III, and IV) in the isotherm shown in Fig. 1. In the initial stage of the collapse process (b) we can see 3D centers of a "point" (granular) type nucleated from the supersaturated condensed phase monolayer. The 3D nuclei gradually grow in a lateral direction (c), finally forming a high density of 3D "stripes" (d). The growth of stripes was not observed when the surface pressure was kept nearly constant near the spike of the isotherm. The time required for the formation of individual 3D stripes in the surface-roughening regime was found to be much longer compared to that required for the development of individual cracks in a random crack network pattern under the same barrier compression conditions. From Figs. 2 and 3, it is evident that the time and spatial scales by which the growth of individual cracks is dominated are somwhat different. This means that the nucleation and growth modes for crack development in each regime become quite different. In other words, the differences in the time and length scales that

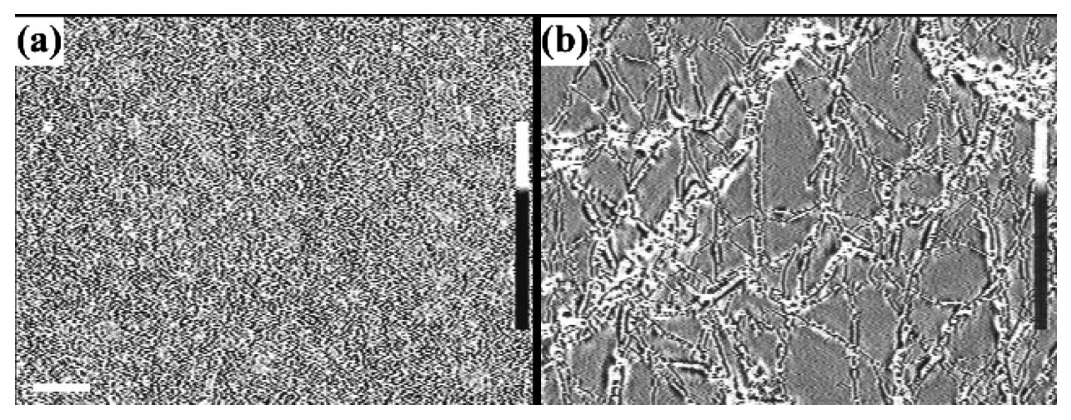

FIG. 2. A surface-roughening crack pattern [(a) $p \mathrm{H}=7.5]$ and a random crack network pattern [(b) $p \mathrm{H}=7.8]$ in stearic acid Langmuir monolayers. These patterns occurred at a late stage of the collapse process above the collapse pressure $\pi_{c}$ in each curve of Fig. 1. The scale bar represents $100 \mu \mathrm{m}$. 


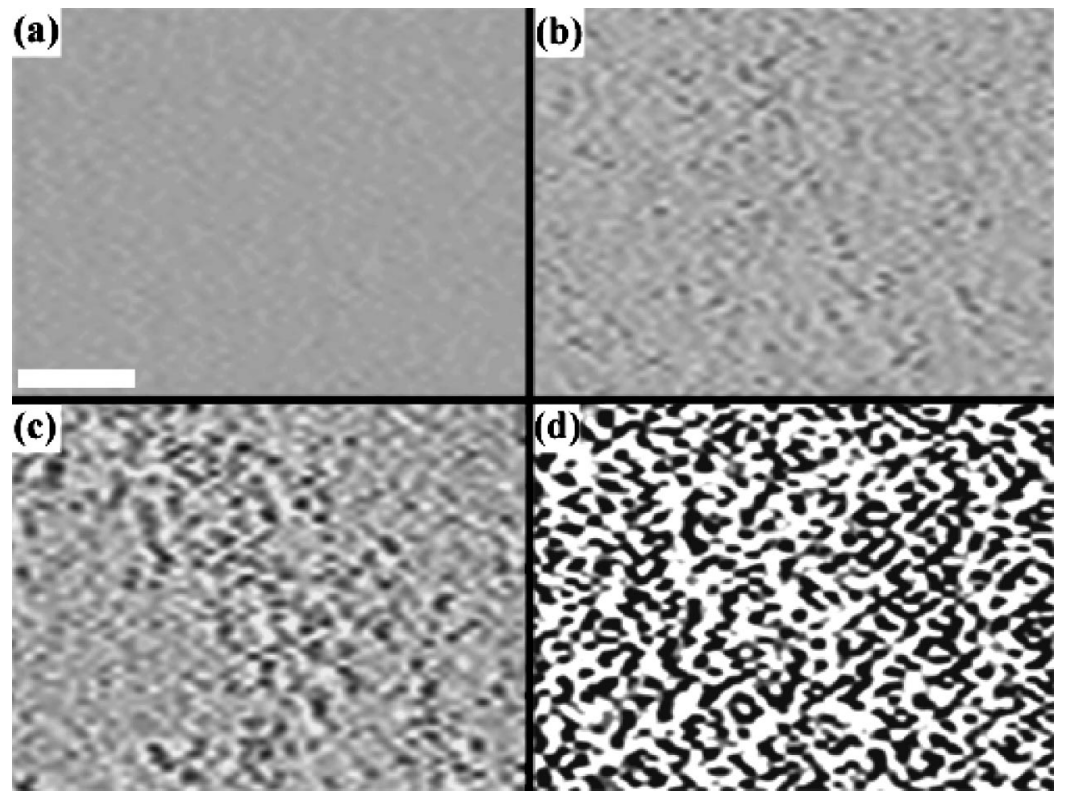

FIG. 3. Evolution of a surface-roughening collapse pattern in a stearic acid Langmuir monolayer at $p \mathrm{H}=7.5$. The images (a), (b), (c), and (d) were taken at the points I, II, III, and IV of the isotherm (a) shown in Fig. 1. The scale bar represents $50 \mu \mathrm{m}$. characterize the two types of crack pattern should reflect the differences in the manner in which the stress stored in the monolayers is released. In this respect, we can say that the release of energy stored in a monolayer characterized by surface-roughening cracking is local, while that in the random crack network is nonlocal. It is clear that surface roughening collapse belongs to the slow collapse regime. Such a fine granular pattern is expected to be observed in monolayers where there is a high nucleation rate and a low growth rate of the $3 \mathrm{D}$ phase, otherwise the occurrence of fast longrange cracking would be realized.

The expected $\mathrm{pKa}$ for a stearic acid monolayer is 5.6 [24]. The degree of ionization of the head group in $p \mathrm{H} 7.5$ and 7.8 is estimated to be $98.5 \%$ and $98.8 \%$, respectively, with the pKa value and the surface potential changing continuously throuoghout the $\mathrm{pH}$ range, indicating gradual dissociation [25]. In spite of these facts, the difference in the degree of ionization of the head group clearly leads to two very different routes to collapse in the system. One might ask, why is the route to collapse so different when the head-group dissociation only differs by $0.3 \%$, although any other variable has been unchanged other than the $p \mathrm{H}$ for the two experiments? In the previous collapse experiments on stearic acid monolayers in the presence of $\mathrm{Co}^{2+}$ ions, the qualitatively different collapse morphology was observed on passing a critical $p \mathrm{H}$ value [18], and the crack coalescence rate in modulation crack evolutions was found to be very sensitive to the $p \mathrm{H}$ values [19]. Thus, it is not unusual that the slight changes of $p \mathrm{H}$ values cause drastic effects to the collapse behavior in Langmuir monolayers. In relation to this, slight changes of the head-group dissociation were observed to cause drastic changes in some properties of Langmuir monolayers and multilayer Langmuir-Blodgett (LB) films of fatty acids $[26,27]$. A discontinuous change of the position correlation length, which is a measure of the lateral attractive interactions between the moleculaes in the monolayer was observed in arachidic acid monolayers in a narrow $p \mathrm{H}$ region [26]. The transitions between alkyl chain tilt angles occurred in
LB films of zinc arachidate through a narrow $p \mathrm{H}$ window [27]. The origins for the sudden changes in the properties were ascribed to the competition between hydrogen bonding and electrostatic repulsive interactions and the extent of metal ion complexation with the acid head group, respectively. The two remarkably different collapse morphologies in our present case might thus be caused by different surface phases from which the $3 \mathrm{D}$ phases occur and/or the different bonding behavior between the molecules which constitute the monolayer. GIXD studies have not been done on monolayers in the $p \mathrm{H}$ and pressure regions under which this study was made and the structural identification of the monolayers that cause the two distinct collapse patterns remains open. Moreover, we will need to tackle to a difficult, nonlinear problem of fracture physics to fully understand the morphological transition and the underlying crack dynamics. We also obtained a similar surface roughening $(p \mathrm{H}=6.5)$ to random network $(p \mathrm{H}=7.0)$ collapse transition in stearic acid monolayers in the presence of $1 \mathrm{mMol} \mathrm{Mn}^{2+}$ ions. The $\mathrm{pKa}$ value was reported to be 6.2 [28].

Below we focus our attention on the topological features of collapsed monolayers in the surface-roughening regime (Fig. 4). Figure 4(a) shows an area of a given film and three different areas in the image enlarged to illustrate the features of the pattern are shown in Figs. 4(b)-4(d). A common feature of the three images is the prevalence of a high density of nearly uniform 3D stripes (of finite length). There is a characteristic length, which is approximately 5-10 $\mu \mathrm{m}$, for the width of each stripe and the nearest-neighbor spacing. The stripes do not extend in a straight line but are often highly curved. This pattern is also characterized by the emergence of partially aligned stripe clusters (modulated with three to ten periods), as shown in Figs. 4(e) and 4(f), which were taken from 4(c) and 4(d), respectively. The local stripe clusters have a characteristic orientational order (i.e., eightfold azimuthal symmetry) as illustrated by the azimuthal modulation in the autocorrelation spectra shown in Figs. 4(b)-4(d), 


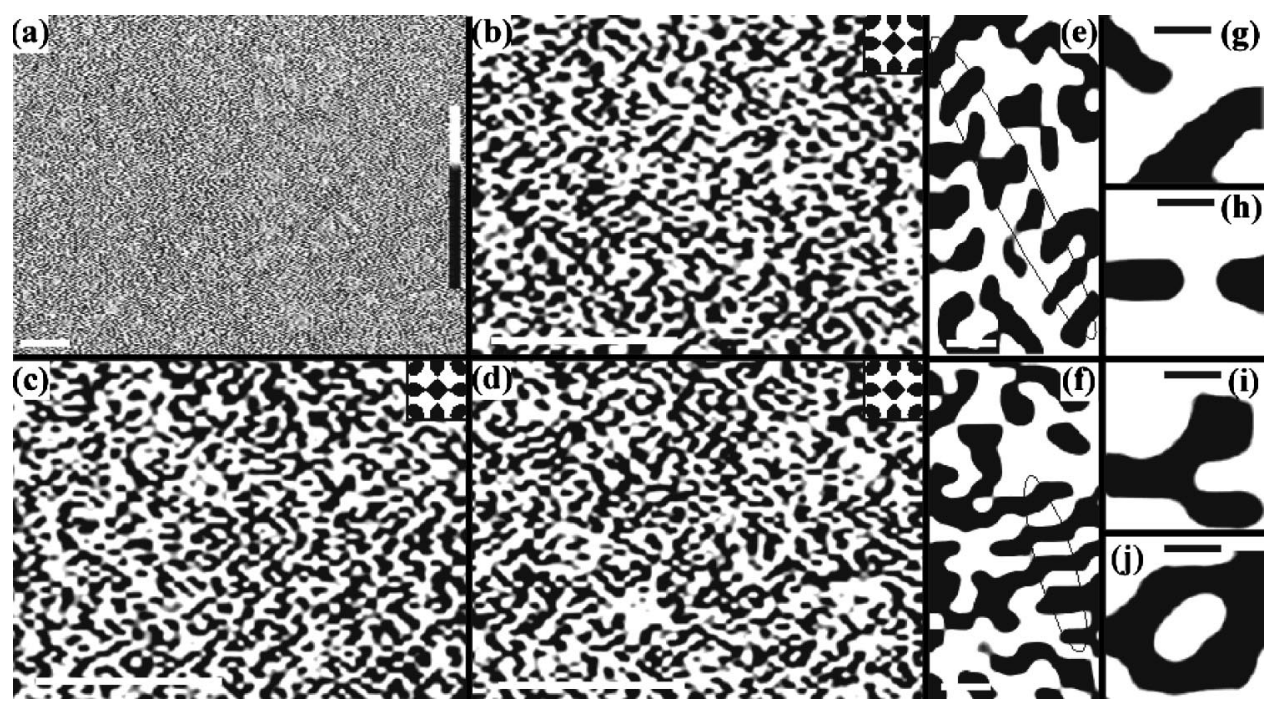

FIG. 4. Stripe patterns observed at a late stage of the collapse process of a stearic acid Langmuir monolayer at $p \mathrm{H}=7.5$. The bars represent $100 \mu \mathrm{m}[(\mathrm{a})-(\mathrm{d})], 10 \mu \mathrm{m}[(\mathrm{e})$ and (f)], and $5 \mu \mathrm{m}[(\mathrm{g})-(\mathrm{j})]$. The image (a) was taken at the location (IV) in the isotherm of Fig. 1(a). The images (b)-(d) are shown enlarged from three different areas of (a). The autocorrelation spectra [shown in the upper right corner in the images (b)-(d)] were taken at the center of each image. Note that the shape of each autocorrelation spectra was independent of the position of the image at which the spectra were taken. The images (e) and (f) [which were taken from (c) and (d), respectively] show examples of partially aligned stripe clusters (enclosed by ellipses). (g)-(j) show constituent elements from a stripe pattern: (g) a single end, (h) twin ends, (i) a branch, (j) a loop.

which were taken at the center of each image, but which were independent of the location at which they were taken, and are essentially those of a partially ordered, stripe phase (however, the pattern is still globally disordered). Both the breaking of rotational symmetry in the growth of stripes and the orientational correlation in the stripe clusters might be assisted by a uniaxial barrier compression. Generally the competition between short-range attractive and long-range repulsive forces leads to periodically modulated phases such as stripes and bubbles [29]. In earlier work stripe phases in Langmuir monolayers were found in the two coexisting fluid phases of a phospholipid monolayer with a small amount of cholesterol [30], or a monolayer containing a binary mixture of phospholipid and cholesterol [31] or in the LE-gas $(G)$ phase coexistence region of one-component Langmuir monolayers [32]. The stripe pattern presented here is unique in that it results from irreversible fracture into the thrid dimension, in contrast to the in-plane growth of the stripe patterns cited above. One more significant feature in the pattern is that the evolution of the collapse pattern in the surfaceroughening regime is dominated by topological constraints in the direction of nucleation and growth of the 3D bulk and that the pattern includes a high density of topological defects in the form of single open ends [where one open end meets one side of the walls of one of the neighboring stripes $(\mathrm{g})]$, twin ends [where two single open-ended stripes meet (h)], branches [where three stripes join at a vertex (i)], and loops (j). The occurrence of these defects breaks the orientational order in the local alignment of stripes. Such a growth instability of 3D stripe domains leads to the condensation of a characteristic stripe phase. Topological defects have been observed in various ordered media such as magnetic films and liquid crystals and play an essential role in the mechanical stability of the phases $[33,34]$. Defects similar to those in our stripe phase have also been observed in self-assembled films of diblock copolymers [35,36]; however, they have not been met in the context of soft matter fractures including Langmuir monolayer collapse as far as we know. There are intriguing questions concerning the origin of the prevalence of individual stripes in the phase and the analysis of their dynamics. It is well known that the different deformation modes of monolayers generally lead to the corresponding dynamic responses [37]. It is thus of interest to consider the shapes of individual stripes and their growth kinetics when a significant amount of shear deformation is added to the collapse kinetics of the monolayer, especially, in the slow collapse limit. In relation to this, it is significant to note that shear deformation of a monolayer with a finite shear modulus is generally represented by a superposition of pure compression with the compression axis at $45^{\circ}$ with respect to the force gradient and a pure rotation, different from a nonrotational uniaxial compression. The barrier compression is uniaxial (from left to right in the images) in our case, and if a principal shear force operates in this direction, the resultant dominant force gradient would be vertical to it. From this viewpoint, the overall eightfold rotational symmetry observed in the surface-roughening pattern suggests the existence of a significant amount of shear deformation in the slow collapse process. It is thus anticipated that a sufficiently large shear deformation will couple to the surfaceroughening morphology of the monolayer. In relation to this, solid phase $S$ of a docosanoic acid monolayer was studied under the application of extensional and shear deformations [38]. It was concluded that the distortion of this phase was accompanied by the propagation of "shear" bands at $\pm 45^{\circ}$ to the principal axis of strain. The authors did not observe morphologies beyond the collapse pressure. We must be aware of the fact that the presence of the vertical walls of the 
trough perpendicular to the barrier motion may provide a significant contribution to the generation of uniaxial shear forces on the monolayers. A different barrier compression mode such as four-barrier compression may cause a qualitatively different collapse pattern. The existence of a rotational component and inhomogeneity in the shear deformation on the monolayer could contribute to the broadening of the spots in the autocorrelation function as well as the appearance of various stripe shapes. The coupling of such shear deformation with the growth directions of $3 \mathrm{D}$ aggregates would thus be possible in the surface-roughening regime due to its slow growth nature and as a result, it would lead to the emergence of the stripe phase with locally preferred directions. The effective coupling of such deformation with the crack directions would not be expected due to the large difference between the relevant time scales in the fast collapse regime [Fig. 1(b)]. From the above considerations, shear deformation of a monolayer during slow collapse could be a significant contributory factor for the stripe pattern observed here, but a detailed analysis of their growth kinetics, however, remains to be done.

\section{CONCLUSIONS}

At the collapse transition in Langmuir monolayers, the surface-roughening crack pattern is manifested by the emergence of a 3D disordered stripe phase. This phase is characterized by (1) the creation of locally aligned stripe clusters, (2) the existence of a characteristic length scale for the stripe width and the spacing between the neighboring stripes, and (3) the inclusion of a high density of topological defects. The coupling of a shear force caused by uniaxial barrier compression and the slow growth of 3D aggregates driven by monolayer cracking might provide a significant contribution to the formation of the characteristic stripe phase observed here.

\section{ACKNOWLEDGMENT}

One of the authors (E.H.) acknowledges Th. M. Fischer for his valuable comments.
[1] V.M. Kaganer, H. Möhwald, and P. Dutta, Rev. Mod. Phys. 71, 779 (1999).

[2] K. Kjaer, J. Als-Nielsen, C.A. Helm, P. Tippman-Krayer, and H. Möhwald, J. Phys. Chem. 93, 3200 (1989).

[3] R.M. Kenn, C. Böhm, A.M. Bibo, I.R. Peterson, H. Möhwald, J. Als-Nielsen, and K. Kjaer, J. Chem. Phys. 95, 2092 (1991).

[4] D.K. Schwartz and C.M. Knobler, J. Phys. Chem. 97, 8849 (1993).

[5] B. Fischer, M.-W. Tsao, J. Ruiz-Garcia, Th.M. Fischer, D.K. Schwartz, and C.M. Knobler, J. Phys. Chem. 98, 7430 (1994).

[6] D. Hönig and D. Möbius, J. Phys. Chem. 95, 4590 (1991).

[7] S. Henon and J. Meunier, Rev. Sci. Instrum. 62, 936 (1991).

[8] Micelles, Monolayers, and Biomembranes, edited by M.N. Jones, D. Chapman, and M. N. Jones (Wiley, New York, 1994).

[9] J.A. Clements, Physiologist 5, 11 (1962).

[10] R.D. Smith and J.C. Berg, J. Colloid Interface Sci. 74, 273 (1980).

[11] H.E. Ries, Jr., Nature (London) 281, 287 (1979).

[12] C. MacFate, D. Ward, and J. Olmsted, Langmuir 9, 1036 (1993).

[13] K.S. Birdi and D.T. Vu, Langmuir 10, 623 (1994).

[14] E. Hatta, H. Hosoi, H. Akiyama, T. Ishii, and K. Mukasa, Eur. Phys. J. B 2, 347 (1998).

[15] H.E. Ries, Jr. and H.W. Swift, Langmuir 3, 353 (1987).

[16] C. Ybert, W. Lu, G. Möller, and C.M. Knobler, J. Phys. Chem. B 106, 2004 (2002).

[17] A. Gopal and K.Y.C. Lee, J. Phys. Chem. B 105, 10348 (2001).

[18] E. Hatta, J. Nagao, and D. Suzuki, Eur. Phys. J. B 11, 609 (1999).

[19] E. Hatta and Th.M. Fischer, J. Phys. Chem. B 106, 589 (2002).

[20] A. Saint Jalmes and F. Gallet, Eur. Phys. J. B 2, 489 (1998); A. Saint Jalmes, F. Gallet, and B. Houchmandzadeh, Europhys. Lett. 28, 565 (1994).

[21] L. Bourdieu, J. Daillant, D. Chatenay, A. Braslau, and D. Colson, Phys. Rev. Lett. 72, 1502 (1994).
[22] M.M. Lipp, K.Y.C. Lee, D.Y. Takamoto, and J.A. Zasadzinski, Phys. Rev. Lett. 81, 1650 (1998).

[23] H. Hosoi, H. Akiyama, E. Hatta, T. Ishii, and K. Mukasa, Jpn. J. Appl. Phys., Part 1 36, 6927 (1997).

[24] J.J. Betts and B.A. Pethica, Trans. Faraday Soc. 52, 1581 (1956).

[25] J.A. Spink, J. Colloid Sci. 18, 512 (1963).

[26] R. Johann, G. Brezesinski, D. Vollhardt, and H. Möhwald, J. Phys. Chem. B 105, 2957 (2001).

[27] A. Dhanabalan, N.P. Kumar, S. Major, and S.S. Talwar, Thin Solid Films 327-329, 787 (1998).

[28] J. Peltonen, M. Linden, H. Fagerholm, E. Gyorvary, and F. Eriksson, Thin Solid Films 242, 88 (1994).

[29] M. Seul and D. Andelman, Science 267, 476 (1995).

[30] R. Weis and H.M. McConnell, J. Phys. Chem. 89, 4453 (1985).

[31] P. Rice and H.M. McConnell, Proc. Natl. Acad. Sci. U.S.A. 86, 6445 (1989); M. Seul and V.S. Chen, Phys. Rev. Lett. 70, 1658 (1993).

[32] K. To, S. Akamatsu, and F. Rondelez, Europhys. Lett. 21, 343 (1993).

[33] M. Kleman, Points, Lines and Walls, in Liquid Crystals, Magnetic Systems, and Various Ordered Media (Wiley, New York, 1983).

[34] P.M. Chaikin and T.C. Lubensky, Principles of Condensed Matter Physics (Cambridge University Press, Cambridge, 1995).

[35] J. Hahm, W.A. Lopes, H.M. Jaeger, and S.J. Sibener, J. Chem. Phys. 109, 10111 (1998).

[36] C. Harrison, Z. Cheng, S. Sethuraman, D.A. Huse, P.M. Chaikin, D.A. Vega, J.M. Sebastian, R.A. Register, and D.H. Adamson, Phys. Rev. E 66, 011706 (1994).

[37] D.A. Edwards, H. Brenner, and D.T. Wasan, Interfacial Transport Processes and Rheology (Butterworth-Heinemann, Boston, 1991).

[38] T. Maruyama, J. Lauger, G.G. Fuller, C.W. Frank, and C.R. Robertson, Langmuir 14, 1836 (1998). 